\title{
Effect of Adaptive Cruise Control on Mixed Traffic Flow: A Comparison of Constant Time Gap Policy with Variable Time Gap Policy
}

\author{
Jiakuan Dong $\mathbb{D}^{D}$, Jiangfeng Wang $\mathbb{D}^{\text {, }}$, Lei Chen, Zhijun Gao, and Dongyu Luo \\ Key Laboratory of Transport Industry of Big Data Application Technologies for Comprehensive Transport, Ministry of Transport, \\ Beijing Jiaotong University, Beijing 100044, China \\ Correspondence should be addressed to Jiangfeng Wang; wangjiangfeng@bjtu.edu.cn
}

Received 1 May 2021; Accepted 30 June 2021; Published 10 July 2021

Academic Editor: Elżbieta Macioszek

Copyright ( 92021 Jiakuan Dong et al. This is an open access article distributed under the Creative Commons Attribution License, which permits unrestricted use, distribution, and reproduction in any medium, provided the original work is properly cited.

With the emerging application of low-level driving automation technology, heterogeneous traffic flow mixed with human-driven vehicles and low-level autonomous vehicles is dawning. In this context, it is imperative to investigate its effect on mixed traffic flow. As a key component for adaptive cruise control (ACC) which is a practical low-level application of driving automation, the time gap policy determines the dynamic of ACC-equipped vehicles and plays a crucial role in traffic flow stability and efficiency. There are two main time gap policies used for ACC at present, namely, constant time gap (CTG) policy and variable time gap (VTG) policy. In this study, we carried out a detailed comparison between these time gap policies to investigate their potential effect on mixed traffic flow, where the analytical- and simulation-based approaches are both considered. Analytical results show that VTG policy is superior to CTG policy in stabilizing the mixed traffic flow. In addition, numerical simulations are also conducted and simulation results further support the analytical results. As for throughput, there is no difference between CTG policy and VTG policy in analytical progress when the same time gap is set at the equilibrium. However, simulation results based on an on-ramp scenario show that the throughput of mixed traffic flow with VTG policy is slightly higher than that of CTG policy. Meanwhile, the scatter of mixed traffic flow with VTG policy in the flow-density diagram gradually clusters in the middle range of density (i.e., $20-40 \mathrm{veh} / \mathrm{km}$ ) with the increase of the penetration rates of ACC vehicles, where the traffic flow operates more efficiently. These results indicate that VTG policy is better than CTG policy when designing controllers for ACC in the context of traffic flow operation and control.

\section{Introduction}

With the development of high-precision sensing and lowlatency communication technologies, connected and automated vehicles (CAVs) are the most promising approach to improve traffic safety and efficiency. However, Level 4 or higher driving automation is unlikely to be applied widely and commercially in near future. As a low-level application of driving automation, adaptive cruise control (ACC) has been realized and deployed in the vehicle market to help drivers to drive safely and comfortably. ACC system automatically adjusts the vehicle speed according to the predesigned control algorithms and shortens the following gap on the premise of ensuring safety.
The most widely used ACC algorithm in literature is the linear feedback control scheme, where the acceleration of ACC-equipped vehicles is proportional to the deviation from target spacing and the relative speed with the preceding vehicle [1]. The target spacing determines the safe following gap and distinguishes different control algorithms. Based on different target spacing strategies, the ACC control scheme can be divided into constant spacing policy, variable spacing policy with constant time gap (CTG), and variable spacing policy with variable time gap (VTG). For the constant spacing policy, the target spacing is time-invariant, resulting in that it is not adapted to complex and dynamic traffic environments. To surmount this problem, the target spacing in CTG policy is defined as the current speed of ACC 
vehicles multiplied by a predefined constant time gap plus a minimum safe standstill spacing. The PATH program developed a CTG policy for ACC and validated it based on field experimental data, which has been widely used in subsequent studies [2,3]. However, the desired time gap in CTG policy is constant and the target spacing is only related to the current vehicle's speed; the real-time traffic dynamics are not considered. Besides, CTG policy may result in large spacing, hence, decreasing traffic throughput $[4,5]$. To fill this gap, some researchers proposed several time-variant desire time gap policies adapting to the real-time traffic environment [6-8]. In addition, some researchers integrated macroscopic traffic flow parameters, such as jam density and free-flow speed, into the desired time gap function [9-11]. However, it is difficult to obtain these parameters precisely in the field, and the performance of the VTG policy may deteriorate with a biased parameter setting. For more detailed information about different types of spacing policies in ACC, please refer to the expository and survey article [12] and the closely related references therein.

With the rapid development of driving automation technology, heterogeneous traffic flow mixed with humandriven vehicles (HDVs) and ACC vehicles is dawning. In this context, it is imperative to investigate the effect of ACC on traffic flow in heterogeneous traffic environments consisting of both ACC vehicles and HDVs. In literature, the effects of ACC on traffic flow dynamics have been investigated with respect to improving road capacity [13-15], suppressing traffic congestion [16,17], reducing traffic risk [18-20], and promoting sustainable transportation [21-23]. Due to the different modelling approaches and parameters setting, these effects are varying. For instance, some research found that traffic congestion can be eliminated in mixed traffic with a proportion of $20 \%-25 \%$ ACC vehicles $[16,17]$. However, Jerath and Brennan [24] found that although the introduction of ACC-enabled vehicles into the traffic stream may produce higher traffic flows, it also resulted in disproportionately higher susceptibility of the traffic flow to congestion. Moreover, Makridis et al. [25] questioned the common assumption that ACC or other automation technologies necessarily improve traffic flow and increase road capacity. Their test results show that the response time of the ACC controller was in the range of $0.8 \mathrm{~s}-1.2 \mathrm{~s}$, which is similar to what is commonly assumed for human drivers. It should be noted that the findings are influenced by the modelling approach and desired time gap policy, and the setting of the time gap plays a critical role in addressing the effect of ACC vehicles on traffic flow. However, most studies mentioned above have only been carried out considering CTG policy for ACC controllers. So far, very little attention has been paid to the role of VTG policy for ACC on traffic flow dynamics $[10,11,13]$. Besides, macroscopic traffic flow parameters are used in these studies to design a time gap policy for ACC, and only the simulation method is used to evaluate the effect of VTG policy for ACC on traffic flow efficiency. Hence, there is still very little scientific understanding of how VTG policy for ACC affects the traffic flow.

Stability analysis of traffic flow is an effective way to explain the mechanisms underlying the generation and propagation of stop-and-go waves [26-29]. Thus, investigating the stability of mixed traffic flow can play an important role in addressing the issue of how VTG policy for ACC affects the traffic flow. In previous studies, some efforts in the literature have been devoted to the string $[16,30-34]$ and traffic flow $[9,35,36]$ stability of ACC vehicles. The focus of most studies lies in homogeneous vehicular traffic. Several studies have been carried out to explore the stability of mixed traffic flow considering CTG policy for ACC vehicles [32-34]. However, very little studies have been designed to investigate the stability of mixed traffic flow considering a VTG policy for ACC.

Investigating the effect of ACC on mixed traffic flow is imperative before its large-scale deployment. There are two main time gap policies used for ACC in literature, namely, CTG policy and VTG policy. However, previous studies mainly focus on the CTG policy for ACC and investigate its effect on traffic flow dynamics. Too little work has been devoted to the stability and throughput analysis of VTG policy in the mixed traffic flow environment. In addition, it is still unclear which time gap policy is superior in terms of stabilizing mixed traffic flow and improving road capacity analytically. Naturally, the objective of this paper is to address these issues and compare the influence of different time gap policies for ACC on mixed traffic analytically and numerically. A modified VTG policy for a classic linear feedback controller of ACC is firstly illustrated. Besides, the intelligent driver model is considered to capture the dynamic of HDVs. Then, the stability of mixed traffic flow considering different time gap policies is derived analytically. Numerical simulation is also conducted to compare the performance of different time gap policies in suppressing traffic oscillation. Finally, the fundamental diagram of the mixed traffic flow is derived and a hypothetical on-ramp scenario is designed to explore the impact of different time gap policies on the throughput of the bottleneck. The structure of this study is shown in Figure 1.

The main contribution of this paper lies in two points. First, to the best of our knowledge, this is the first attempt to analytically investigate the stability of mixed traffic flow with the VTG policy for ACC. The stability of mixed traffic flow is a fundamental property for different VTG policies, which would affect the efficiency and safety of mixed traffic flow. Using the framework proposed in this study, a more intuitive performance indicator can be used to evaluate new VTG policies in future studies. Second, a detailed comparison between different time gap policies for ACC on the stability and throughput analysis of mixed traffic flow are investigated in both analytical and simulation-based ways. The findings should make an important contribution to the field of ACC controller design and vehicular traffic control.

The remainder of this paper is organized as follows. Section 2 presents the car-following models for ACC with VTG and CTG policy and human-driving vehicles. Then, the string stability criterion of mixed traffic flow is derived in Section 3. Besides, numerical simulations are conducted to investigate the effect of different time gap policies on traffic oscillation mitigation in the same section. Section 4 derives the fundamental diagrams in the mixed traffic flow and 


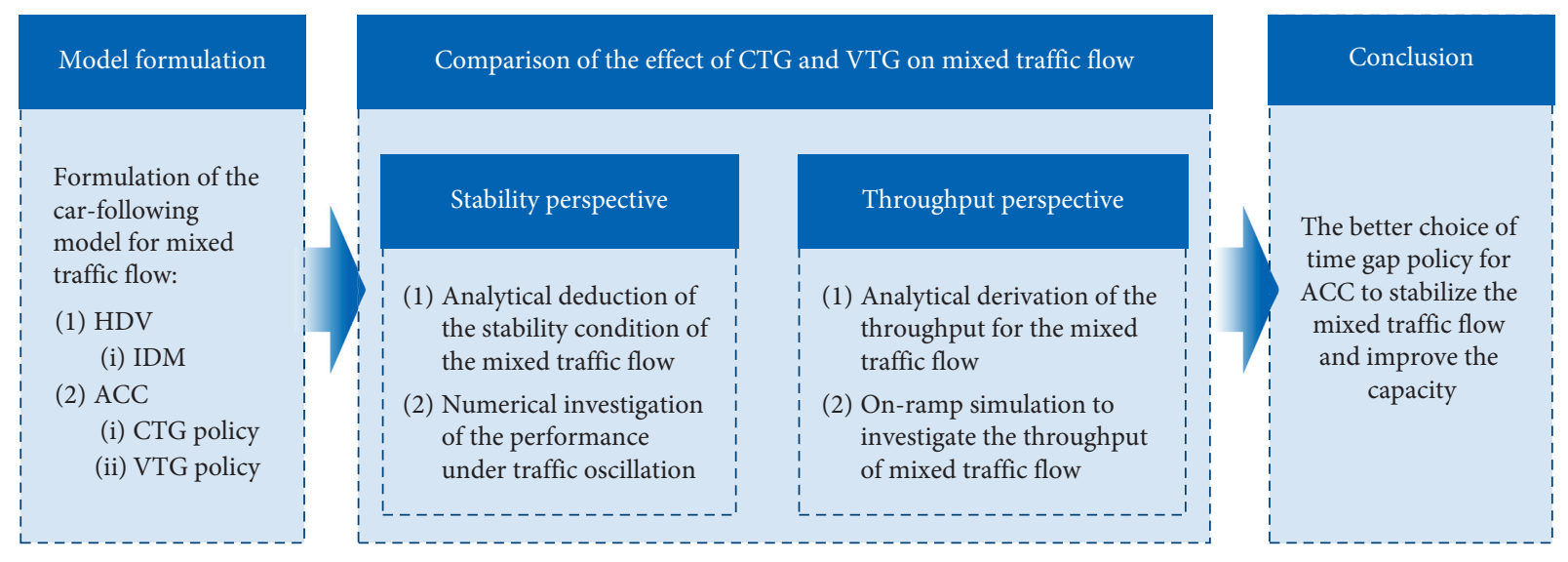

Figure 1: The structure diagram of this study.

conducts on-ramp simulation experiments to verify the analytical analysis results. Finally, conclusions are delivered in Section 5.

\section{Model Formulation}

2.1. Adaptive Cruise Control with Different Time Gap Policies. Currently, the prevalent control structure for ACC is a linear feedback controller, which is shown as

$$
a=k_{1}\left(s-s_{0}-t_{h} * v\right)+k_{2} \Delta v,
$$

where $a$ is the calculated acceleration for ACC vehicles, $s, v$, and $\Delta v$ are distance gap, speed, and relative speed of ego vehicle, respectively, $s_{0}$ represents the standstill distance between successive vehicles, $t_{h}$ is the desired time gap for ACC vehicles, and $k_{1}$ and $k_{2}$ are control gains.

Depending on the choice of specific formulation for $t_{h}$, different time gap policies for ACC can be distinguished. Specifically, $t_{h}$ is a predefined constant for CTG policy, which means that the desired time gap maintains invariant when ACC is in operation. In contrast, for VTG policy, $t_{h}$ is time-variant to acclimate the surrounding traffic dynamics in real-time. In the literature, $t_{h}$ for VTG policy gives the desired time gap as a function of the relative speed. Besides, to ensure traffic safety and efficiency, upper and lower bounds are usually introduced to limit $t_{h}$. Thus, the desired time gap for VTG can be described in the following form:

$$
t_{h}(\Delta v)= \begin{cases}t_{h}^{\min }, & \text { if } \Delta v \geq \Delta v_{\mathrm{ub}}, \\ F(\Delta v), & \text { if } \Delta v_{\mathrm{lb}}<\Delta v<\Delta v_{\mathrm{ub}}, \\ t_{h}^{\max }, & \text { if } \Delta v \leq \Delta v_{\mathrm{lb}}\end{cases}
$$

where $t_{h}^{\max }$ and $t_{h}^{\min }$ are the upper and lower bound for the time gap, respectively. $\Delta v_{\mathrm{ub}}$ and $\Delta v_{\mathrm{lb}}$ represent the upper bound and lower bound of relative speed for the time gap policy. $F(\Delta v)$ is a continuous and monotonically decreasing function. This means that when the relative speed is positive (i.e., the speed of the ego vehicle is smaller than the speed of the preceding), the ego vehicle is expected to accelerate to achieve the same speed as the preceding vehicle by operation in a smaller time gap. Similarly, when the relative speed is positive (i.e., the speed of the ego vehicle is greater than the preceding vehicle), the ego vehicle is expected to decelerate and operate in a larger time gap. Figure 2 shows two possible choices of the time gap policy for VTG. In previous studies $[6,11]$, the linear function of $F(\Delta v)$ is preferred, as shown in Figure 2(a) and the following equation:

$$
F(\Delta v)=t_{0}-c_{h} \Delta v
$$

where $t_{0}$ is a positive constant and $c_{h}$ is a positive coefficient for relative speed. This gradient of the linear function form is discontinuous at the bound points, which may lead to discontinuities in the jerk and hence discomfort the driver. Thus, a smooth nonlinear function form of $F(\Delta v)$ is considered in this paper, as shown in Figure 2(b) and the following equation:

$$
F(\Delta v)=t_{h}^{\max }-\left(\frac{t_{h}^{\max }-t_{h}^{\min }}{2}\right)\left(1-\cos \left(\pi \frac{\Delta v-\Delta v_{\mathrm{lb}}}{\Delta v_{\mathrm{ub}}-\Delta v_{\mathrm{lb}}}\right)\right) .
$$

It is noted that, in this paper, the same modulus of $\Delta v_{\mathrm{ub}}$ and $\Delta v_{\mathrm{lb}}$ is adopted (i.e., $\left|\Delta v_{\mathrm{ub}}\right|=\left|\Delta v_{\mathrm{lb}}\right|=\Delta v_{c}$ ), and three values $(1 \mathrm{~m} / \mathrm{s}, 2 \mathrm{~m} / \mathrm{s}$, and $3 \mathrm{~m} / \mathrm{s})$ are investigated in the following sections.

In the field test conducted by PATH [2], the desired time gap for ACC vehicles is set to $1.1 \mathrm{~s}$. In addition, the optimal values of the control gains in equation (1) are $k_{1}=0.23$ and $k_{2}=0.07$. In this paper, we use the same value settings referring to PATH's field test. However, for VTG policy, a smaller desired time gap of $0.6 \mathrm{~s}$ is set for $t_{h}^{\min } . t_{h}^{\max }$ is determined based on previous studies of PATH [37], in which the acceptance ratios of the desired time gap for ACC vehicles are found to be different among drivers, $31.1 \%$ at $2.2 \mathrm{~s}$, $18.5 \%$ at $1.6 \mathrm{~s}$, and $50.4 \%$ at $1.1 \mathrm{~s}$. Thus, different $t_{h}^{\max }$ are tested in this paper.

2.2. Car-Following Model for Human-Driven Vehicles. In this paper, the intelligent driver model (IDM) $[29,38]$ is selected to model the dynamic of human-driven vehicles. IDM utilizes a continuous function to capture the relation between the following vehicle's acceleration and the local 


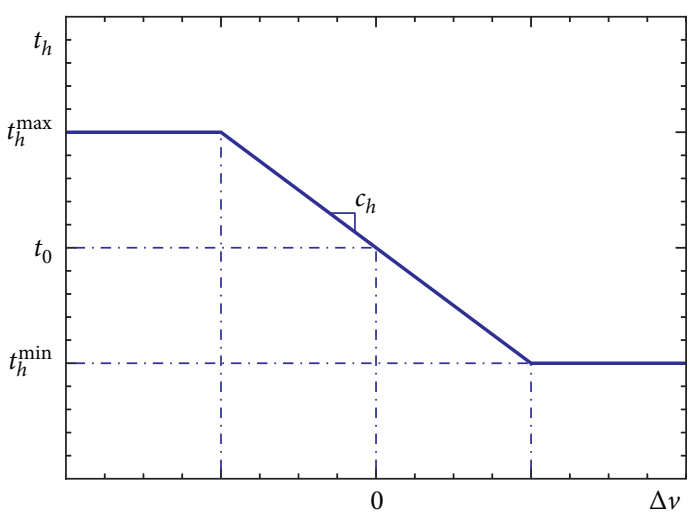

(a)

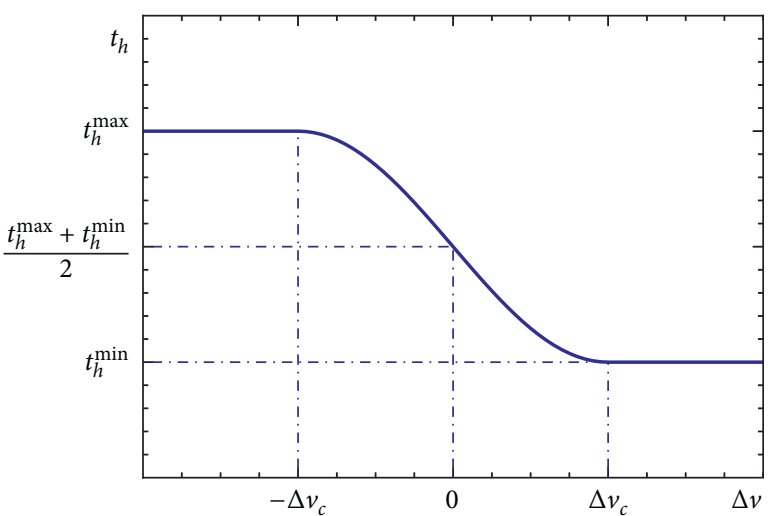

(b)

Figure 2: Time gap policy functions. (a) Function form corresponds to equation (3). (b) Function form corresponds to equation (4).

dynamic information (i.e., following vehicle's speed, spacing, and relative speed), which is shown as

$$
\begin{aligned}
\dot{v}_{n} & =a\left[1-\left(\frac{v_{n}}{v_{0}}\right)^{4}-\left(\frac{s^{*}\left(v_{n}, \Delta v_{n}\right)}{s_{n}}\right)^{2}\right], \\
s^{*}\left(v_{n}, \Delta v_{n}\right) & =s_{0}+T v_{n}-\frac{v_{n} \Delta v_{n}}{2 \sqrt{a b}},
\end{aligned}
$$

where Table 1 summarizes the parameters of IDM used in this paper.

The materials and methods section should contain sufficient detail so that all procedures can be repeated. It may be divided into headed subsections if several methods are described.

\section{String Stability Analysis}

3.1. Analytical Investigation of String Stability. Referring to [29], the dynamic of car-following behavior with an explicit acceleration function can be formulated by the following coupled differential equations:

$$
\left\{\begin{array}{l}
\dot{x}_{n}=v_{n} \\
\dot{v}_{n}=f\left(s_{n}, \Delta v_{n}, v_{n}\right)
\end{array}\right.
$$

The equilibrium of the car-following model is characterized by the same speed for all vehicles (i.e., $v_{n}=v_{e}$ ) and no accelerations (i.e., $a_{n}=0$ ). Substituting these conditions into equation (8), we can get a function $v_{e}=V\left(s_{e}\right)$ that satisfies $\dot{v}_{n}=f\left(s_{e}, 0, V\left(s_{e}\right)\right)=0$ for all $s_{e}>0 . s_{e}$ is called the equilibrium gap, and the steady-state equilibrium can be specified by $\left(s_{e}, v_{e}\right)$.

The stability of an equilibrium determines whether the traffic remains nearby, gets closer, or moves further away from the original equilibrium after a perturbation. String stability is defined based on the propagation of fluctuation in one vehicle's dynamics to the upstream traffic. Considering an equilibrium $\left(s_{e}, v_{e}\right)$ for a given car-following model, if the perturbation decays as it propagates upstream, it is string stable for this car-following model at the current equilibrium. Otherwise, it is string unstable.
TABle 1: Parameters of IDM used in this paper.

\begin{tabular}{lcc}
\hline Parameter & Value & Description \\
\hline$T$ & $1.1 \mathrm{~s}$ & Desired time gap \\
$a$ & $1.0 \mathrm{~m} \cdot \mathrm{s}^{-2}$ & Desired acceleration \\
$b$ & $2.0 \mathrm{~m} \cdot \mathrm{s}^{-2}$ & Maximum comfortable deceleration \\
$L$ & $5 \mathrm{~m}$ & Vehicle length \\
$s_{0}$ & $2 \mathrm{~m}$ & Minimum safety gap \\
$v_{0}$ & $33.33 \mathrm{~m} \cdot \mathrm{s}^{-1}$ & Desired speed \\
\hline
\end{tabular}

3.2. Homogenous Traffic Flow. There are abundant studies on the string stability of car-following models [39]. Among them, Wilson [26] normalized the coupled differential equations of the car-following model and gave the linear string unstable condition of homogeneous traffic flow:

$$
\frac{1}{2}\left(f_{v}\right)^{2}-f_{\Delta v} f_{v}-f_{s}<0
$$

where $f_{v}, f_{\Delta v}$, and $f_{s}$ are the partial derivatives of the carfollowing model $f$ with respect to speed, relative speed, and distance gap at equilibrium $\left(s_{e}, v_{e}\right)$, respectively.

When traffic is at equilibrium, combining $\Delta v=0$ with equation (4) leads to

$$
\left\{\begin{array}{c}
t_{h}\left(s_{e}, v_{e}\right)=\frac{t_{h}^{\max }+t_{h}^{\min }}{2}, \\
\frac{\mathrm{d} t_{h}}{\mathrm{~d} \Delta v}\left(s_{e}, v_{e}\right)=-\frac{\left(t_{h}^{\max }-t_{h}^{\min }\right) \pi}{4 \Delta v_{c}} .
\end{array}\right.
$$

Then, the three partial derivatives of the ACC vehicle with VTG policy can be obtained:

$$
\left\{\begin{array}{l}
f_{v}^{\mathrm{VTG}}=-k_{1} \frac{t_{h}^{\max }+t_{h}^{\min }}{2} \\
f_{\Delta v}^{\mathrm{VTG}}=k_{2}+k_{1} v_{e} \frac{\left(t_{h}^{\mathrm{max}}-t_{h}^{\mathrm{min}}\right) \pi}{4 \Delta v_{c}} \\
f_{s}^{\mathrm{VTG}}=k_{1}
\end{array}\right.
$$


Substituting equation (10) into equation (8), we obtain the instability condition of the ACC vehicle with the proposed VTG policy:

$$
\frac{\left(t_{h}^{\max }+t_{h}^{\min }\right)^{2}}{8} k_{1}^{2}+\frac{t_{h}^{\max }+t_{h}^{\min }}{2} k_{1}\left[k_{2}+k_{1} v_{e} \frac{\left(t_{h}^{\max }-t_{h}^{\min }\right) \pi}{4 \Delta v_{c}}\right]-k_{1}<0 .
$$

For the ACC model with CTG policy, the three partial derivatives are

$$
\left\{\begin{array}{l}
f_{v}^{\mathrm{CTG}}=-k_{1} t_{h}, \\
f_{\Delta v}^{\mathrm{CTG}}=k_{2}, \\
f_{s}^{\mathrm{CTG}}=k_{1},
\end{array}\right.
$$

Substituting equation (12) into equation (8), we obtain the instability condition of the ACC vehicle with CTG policy:

$$
\frac{1}{2}\left(k_{1} t_{h}\right)^{2}+k_{1} k_{2} t_{h}-k_{1}<0
$$

It is worth noting that the stability value of the VTG policy is positively related to the equilibrium speed $v_{e}$. For CTG policy, however, the stability value is a constant after the desired time gap is given. Figure 3 shows the stability value curve at varying equilibrium speeds with CTG and VTG policy for ACC vehicles. As shown in Figure 3, the stability value of the CTG policy is negative constant, which means the homogeneous traffic of ACC vehicles with CTG policy is unstable at all equilibrium. As for VTG policy, the stability value increases as $v_{e}$ increases. Besides, the stability condition is getting better with the setting larger $t_{h}^{\max }$ or smaller $\Delta v_{c}$. This indicates that the design of a larger maximum desired time gap and lower critical relative speed of VTG for ACC vehicles is conducive to stabilizing the homogeneous traffic flow with ACC vehicles.

3.3. Heterogeneous Traffic Flow. Following Wilson's study [26], Ward [40] extended the linear stability analysis to the heterogeneous car-following model situation and gave the linear string unstable condition of heterogeneous traffic flow:

$$
\sum_{N}\left(\left[\frac{1}{2}\left(f_{v}^{n}\right)^{2}-f_{\Delta v}^{n} f_{v}^{n}-f_{s}^{n}\right]\left[\prod_{m \neq n} f_{s}^{m}\right]^{2}\right)<0,
$$

where $n$ and $m$ denote different vehicle types, $N$ is total vehicle number, and $f_{v}, f_{\Delta v}$, and $f_{s}$ are partial derivatives of different car-following models with respect to speed, relative speed, and distance gap, respectively.

From equation (14), it is worth noting that the instability of heterogeneous traffic is only related to the proportion of different vehicle types. Hence, assume there are $N$ vehicles in heterogeneous traffic flow and the proportion of ACC vehicles is $p$. Then, according to equation (14), the linear string instability of the mixed traffic is

$$
\begin{aligned}
& N(1-p)\left[\frac{1}{2}\left(f_{v}^{M}\right)^{2}-f_{\Delta v}^{M} f_{v}^{M}-f_{s}^{M}\right]\left[\left(f_{s}^{M}\right)^{N(1-p)-1}\left(f_{s}^{A}\right)^{N p}\right]^{2} \\
& +N p\left[\frac{1}{2}\left(f_{v}^{A}\right)^{2}-f_{\Delta v}^{A} f_{v}^{A}-f_{s}^{A}\right]\left[\left(f_{s}^{M}\right)^{N(1-p)}\left(f_{s}^{A}\right)^{N p-1}\right]^{2}<0
\end{aligned}
$$

where $M$ and $A$ indicate human-driven vehicles and ACC vehicles, respectively.

Extracting common factors $N$ and $\left[\left(f_{s}^{M}\right)^{N(1-p)}\right.$ $\left.\left(f_{s}^{A}\right)^{N p}\right]^{2}$, equation (15) can be written as

$$
\begin{aligned}
& (1-p)\left[\frac{1}{2}\left(f_{v}^{M}\right)^{2}-f_{\Delta v}^{M} f_{v}^{M}-f_{s}^{M}\right]\left(f_{s}^{M}\right)^{-2} \\
& +p\left[\frac{1}{2}\left(f_{v}^{A}\right)^{2}-f_{\Delta v}^{A} f_{v}^{A}-f_{s}^{A}\right]\left(f_{s}^{A}\right)^{-2}<0 .
\end{aligned}
$$

The partial derivatives of IDM are calculated based on equations (5) and (6):

$$
\left\{\begin{array}{l}
f_{s}^{M}=2 a \frac{\left[1-\left(v_{e} / v_{0}\right)^{4}\right] \sqrt{1-\left(v_{e} / v_{0}\right)^{4}}}{s_{0}+v_{e} T}, \\
f_{v}^{M}=-\frac{4 a v_{e}^{3}}{v_{0}^{4}}-\frac{2 a T\left[1-\left(v_{e} / v_{0}\right)^{4}\right]}{s_{0}+v_{e} T}, \\
f_{\Delta v}^{M}=\sqrt{\frac{a}{b}} \frac{v_{e}\left[1-\left(v_{e} / v_{0}\right)^{4}\right]}{s_{0}+v_{e} T} .
\end{array}\right.
$$

Figure 4 shows the stability analysis results of heterogeneous traffic under varying penetration rates of ACC vehicles. Specifically, Figure 4(a) shows the stability curve of mixed traffic with CTG policy, and Figures 4(b)-4(d) show the stability curve of mixed traffic with varying maximum desired time gap of VTG policy. It is noted that the stability condition of mixed traffic with VTG policy is better than that with CTG policy. Moreover, for VTG policy, with the increase of $t_{h}^{\max }$, the stability condition is getting better gradually. This indicates that using the VTG policy for ACC vehicles is better than the CTG policy in terms of stabilizing mixed traffic flow. In addition, the design of a larger desired time gap $\left(t_{h}^{\max }\right)$ of VTG policy is conducive to stabilizing the mixed traffic flow.

3.4. Numerical Investigation of String Stability. In this section, numerical simulations are conducted to verify the effectiveness of different time gap policies for mitigating traffic oscillations in mixed traffic flow. Following previous studies [41, 42], oscillations can be simulated by producing periodical acceleration/deceleration. Hence, a predesigned oscillation with a period of $4 \mathrm{~s}$ and acceleration/deceleration of $\pm 1 \mathrm{~m} / \mathrm{s}^{2}$ is introduced in the leading vehicle's trajectory file at $40 \mathrm{~s}$. The initial speed of all vehicles is $20 \mathrm{~m} / \mathrm{s}$ and the total oscillation time introduced in the leading vehicle is $20 \mathrm{~s}$. Ten vehicles with different car-following models are set to 


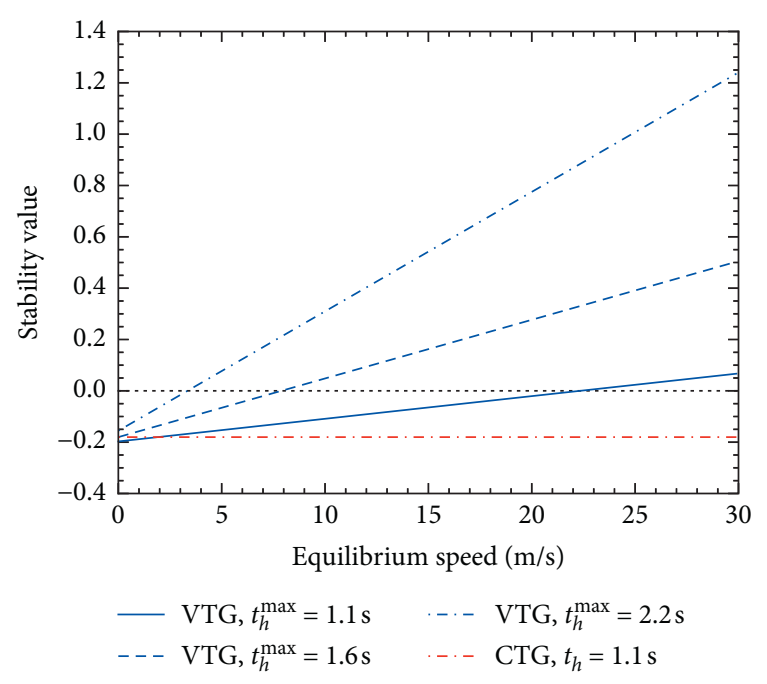

(a)

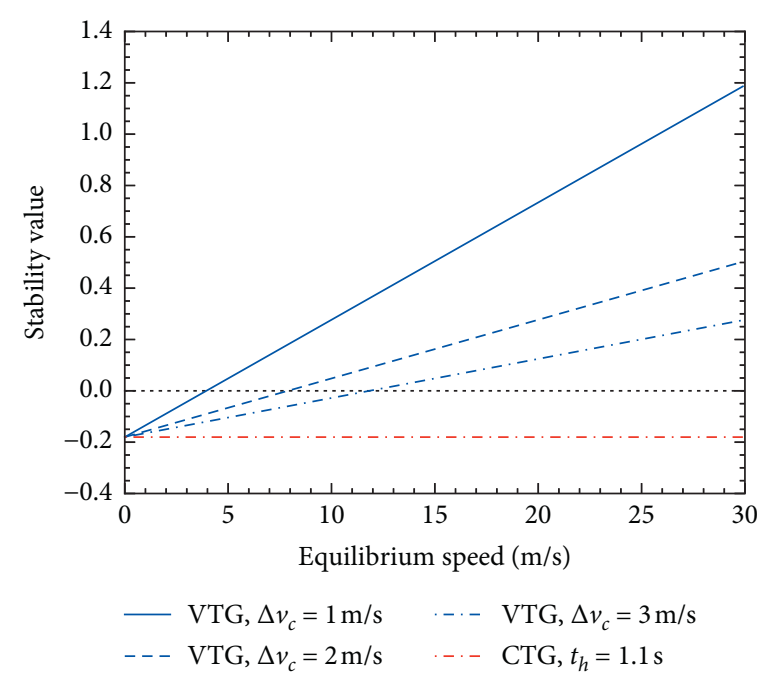

(b)

FIgUre 3: Stability of homogeneous ACC vehicles with different desired time gap policies. (a) Effect of $t_{h}^{\max }$ on stability. (b) Effect of $\Delta v_{c}$ on stability.

follow the leading one. The acceleration, speed, and distance gap between successive vehicles are collected for further analysis. A performance metric named the total oscillation time of the platoon is defined as the time duration for all vehicles in the platoon to return to the initial state after oscillation occurred.

Figure 5 depicts the acceleration, speed, and gap changes of the platoon when following the leading car with the predesigned oscillation, in which the plots of the left column show the case of ACC vehicles with CTG policy, while the plots of the right column are ACC vehicles with VTG policy. The penetration of ACC vehicles is $100 \%$. The leading car is labelled by 0 , and the following cars are labelled from 1 to 10 . Specifically, Figures 5(a) and 5(b) reveal the speed changes under different time gap policies of ACC. As can be seen from Figure 5, the speed of all vehicles in the platoon is back to initial equilibrium speed eventually. However, the speed fluctuation in Figure 5(a) amplifies when approaching the platoon tail and the magnitude of speed fluctuation of the tail vehicle is larger than that of the leading vehicle. Additionally, the speed fluctuation lasts for the following simulation time. In Figure 5(b), the magnitude of speed fluctuation decreases gradually when approaching the platoon tail. Meanwhile, the speed fluctuation of all vehicles returns to the initial equilibrium promptly after the periodic oscillation of the leading vehicle. Similar observations can be made by comparing acceleration changes (cf. Figures 5(c) and 5(d)) and gap changes (cf. Figures 5(e) and 5(f)).

Figure 6 shows the comparison of the total oscillation time of the platoon with different time gap policies in the mixed traffic environment. The penetration rate is from 0 to $100 \%$ with a spacing of $10 \%$. As can be seen from Figure 6, the total oscillation time of the platoon of VTG policy decreases as the penetration rate of ACC increases in mixed traffic. As for mixed traffic with CTG policy, however, the total oscillation time of the platoon soars when the penetration is larger than $30 \%$. It means that mixed traffic flow with VTG policy has a smaller fluctuation time than that with CTG policy; namely, the VTG policy is effective to mitigate traffic oscillations in the mixed traffic environment.

\section{Throughput Analysis}

4.1. Analytical Investigation of Throughput. Referring to [26], when the traffic is at equilibrium, there is no acceleration for any vehicles, and an equilibrium gap-speed mapping function $v_{e}(s)$ can be found, which is also termed the microscopic fundamental diagram. As analysed before, the relative speed and acceleration are both zero at equilibrium (i.e., $\dot{v}=0, \Delta v=0$ ), and the desired time gap of VTG policy is $t_{h}=\left(\left(t_{h}^{\max }+t_{h}^{\min }\right) / 2\right)$. Substituting this information into equation (4), we can obtain the equilibrium speed-gap mapping function $s_{e}(v)$ of VTG policy and CTG policy:

$$
\begin{aligned}
& s_{e}(\mathrm{VTG})=\frac{t_{h}^{\max }+t_{h}^{\min }}{2} v+s_{0}, \\
& s_{e}(\mathrm{CTG})=t_{h} * v+s_{0} .
\end{aligned}
$$

The equilibrium speed-gap mapping function $s_{e}(v)$ of IDM is

$$
s_{e}(v)=\frac{s_{0}+v T}{\sqrt{1-\left(v / v_{0}\right)^{4}}} .
$$

Figure 7 shows the microscopic fundamental diagram of IDM and ACC vehicles with different desired time gap policies. As shown in Figure 7, the microscopic fundamental diagrams of CTG policy with $t_{h}=1.1 \mathrm{~s}$ and VTG policy with $t_{h}^{\max }=1.6 \mathrm{~s}, t_{h}^{\min }=0.6 \mathrm{~s}$ are identical. Additionally, the equilibrium gaps of VTG policy with $t_{h}^{\max }=1.6 \mathrm{~s}, t_{h}^{\min }=0.6 \mathrm{~s}$ and $t_{h}^{\max }=1.1 \mathrm{~s}, t_{h}^{\min }=0.6 \mathrm{~s}$ are no greater than that of IDM in the full range of equilibrium speed. However, for VTG 


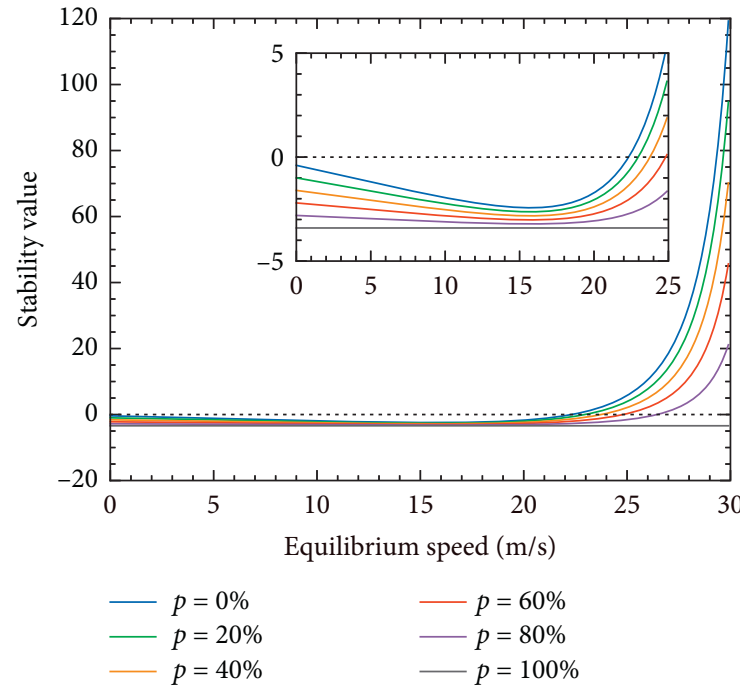

(a)

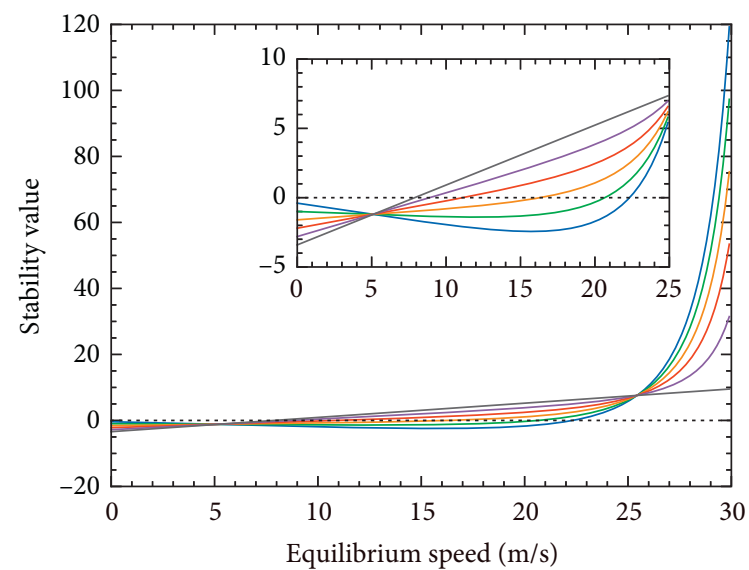

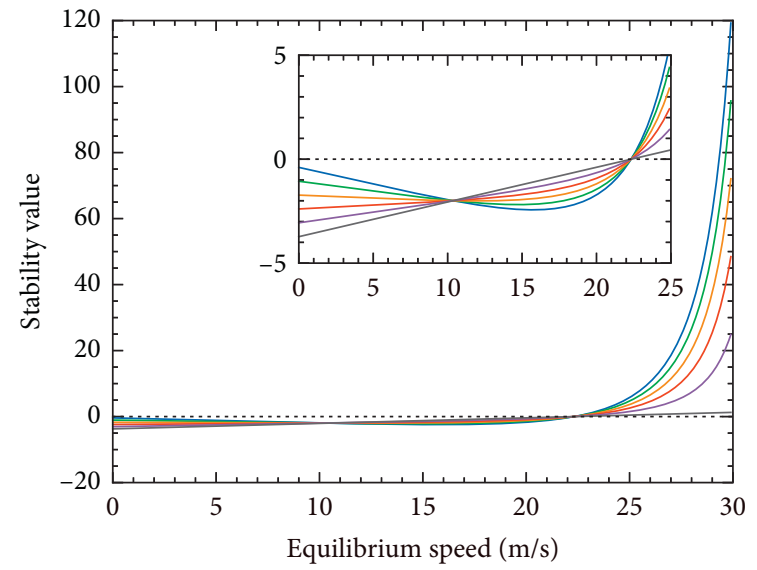

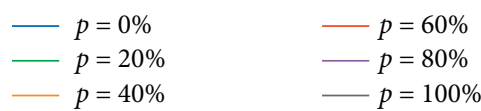

(b)

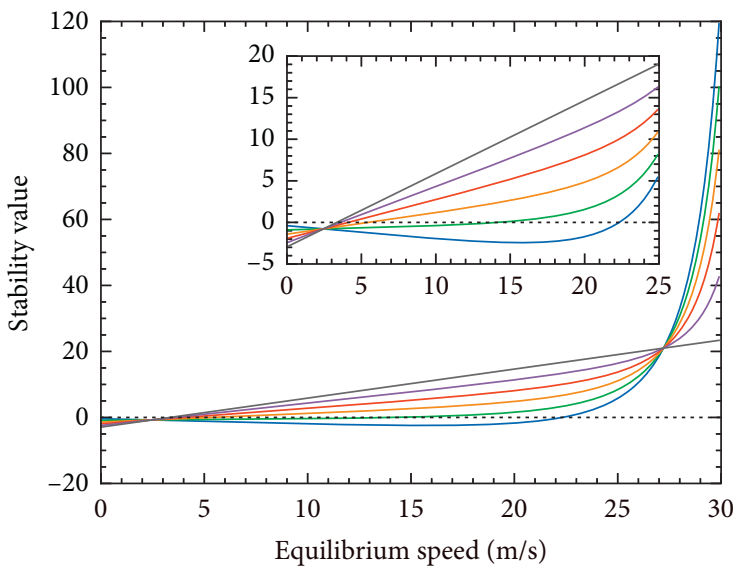

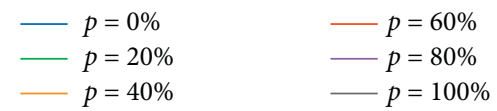

(c)

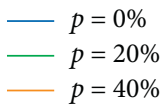$$
\begin{aligned}
p & =60 \% \\
-p & =80 \% \\
p & =100 \%
\end{aligned}
$$

(d)

Figure 4: Stability of heterogeneous traffic flow with different desired time gap policies. (a) CTG policy with $t_{h}=1.1 \mathrm{~s}$. (b) VTG policy with $t_{h}^{\min }=0.6 \mathrm{~s}$ and $t_{h}^{\max }=1.1 \mathrm{~s}$. (c) VTG policy with $t_{h}^{\min }=0.6 \mathrm{~s}$ and $t_{h}^{\max }=1.6 \mathrm{~s}$. (d) VTG policy with $t_{h}^{\min }=0.6 \mathrm{~s}$ and $t_{h}^{\max }=2.2 \mathrm{~s}$.

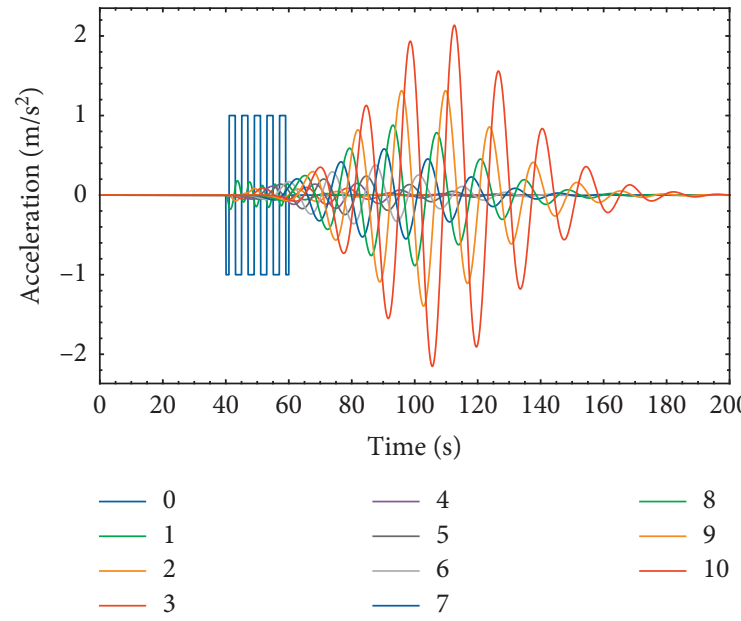

(a)
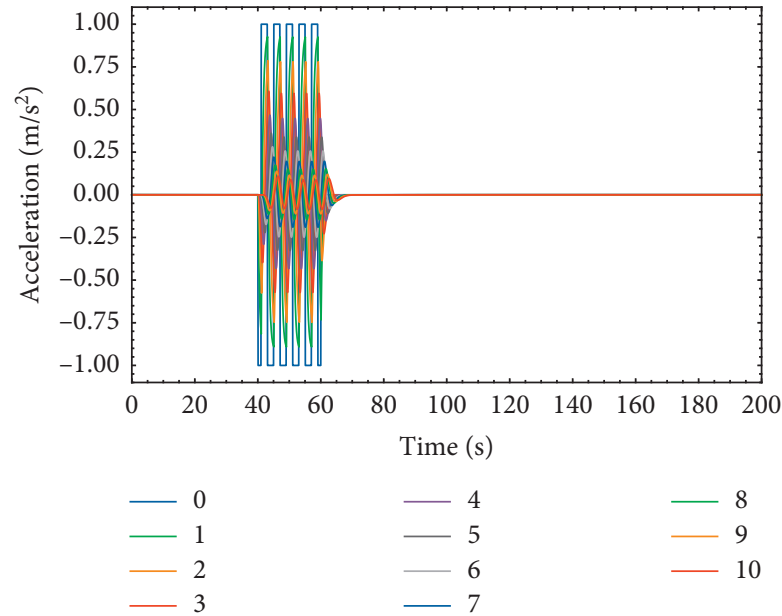

(b)

Figure 5: Continued. 

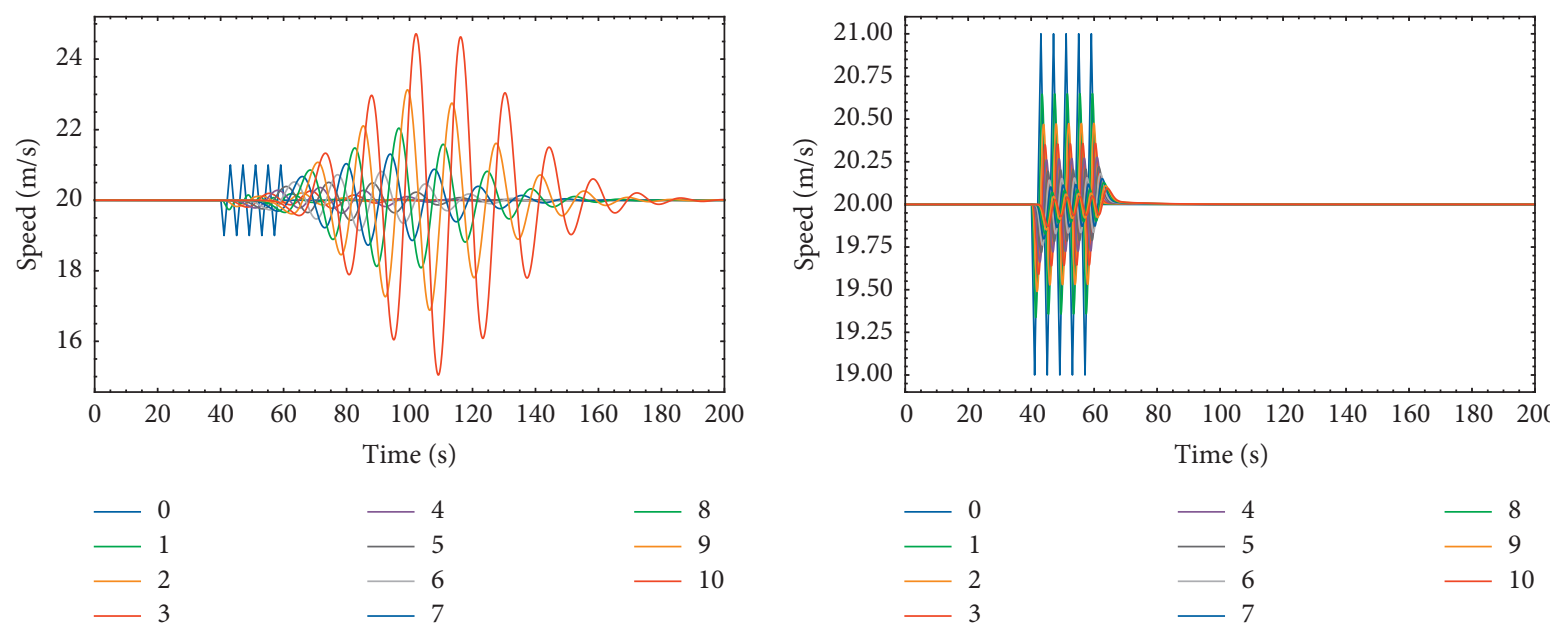

(c)

(d)
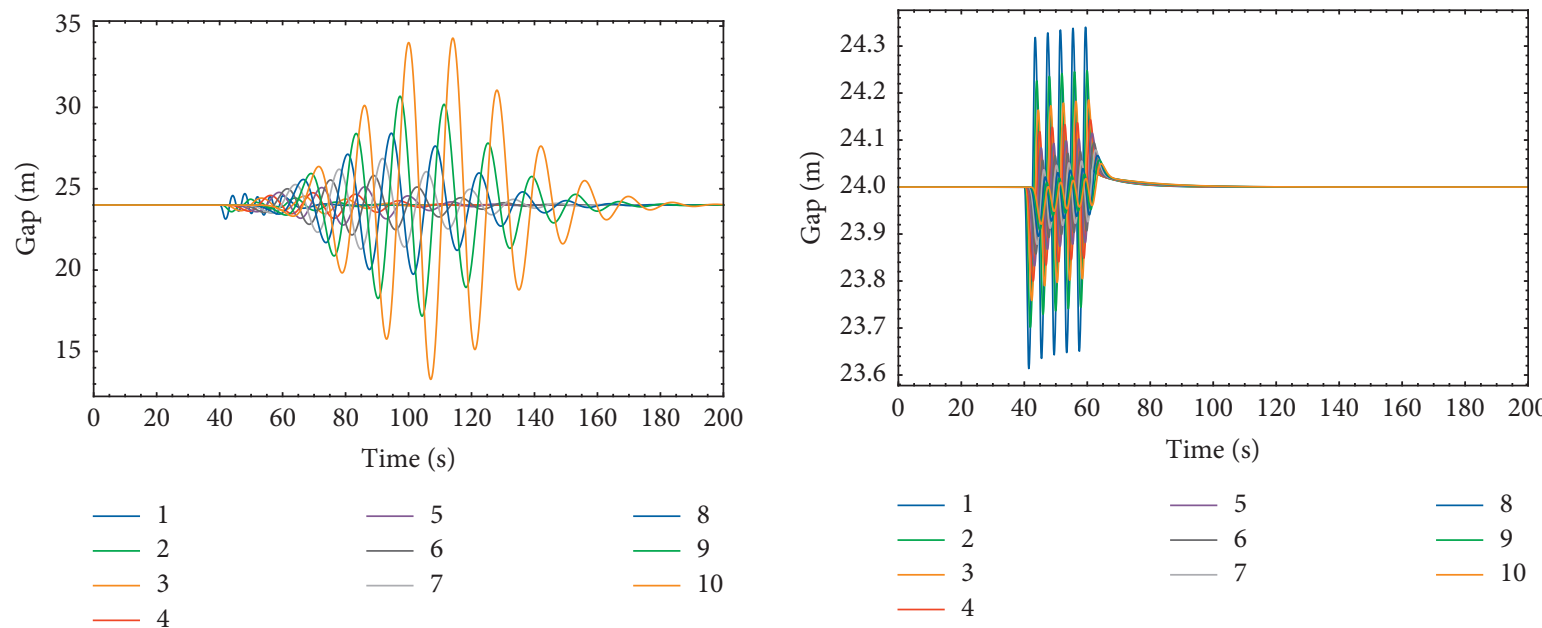

(e)

(f)

FIGURE 5: Platoon dynamic comparison of CTG policy (left column) and VTG policy (right column) in traffic oscillation when penetration of ACC is 1. (a) Time history of acceleration. (b) Time history of acceleration. (c) Time history of speed. (d) Time history of speed. (e) Time history of gap. (f) Time history of gap.

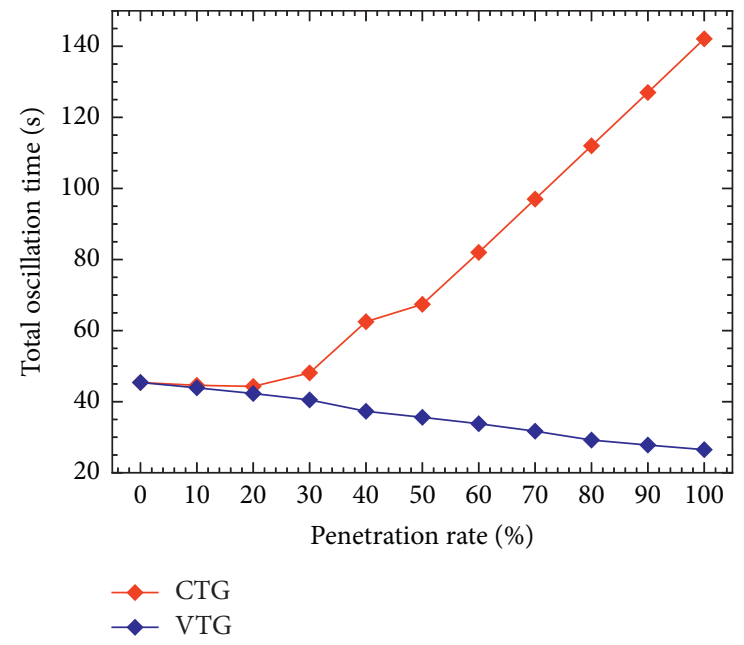

FIgURE 6: Comparison of the total oscillation time for the platoon with different desired time gap policies. 


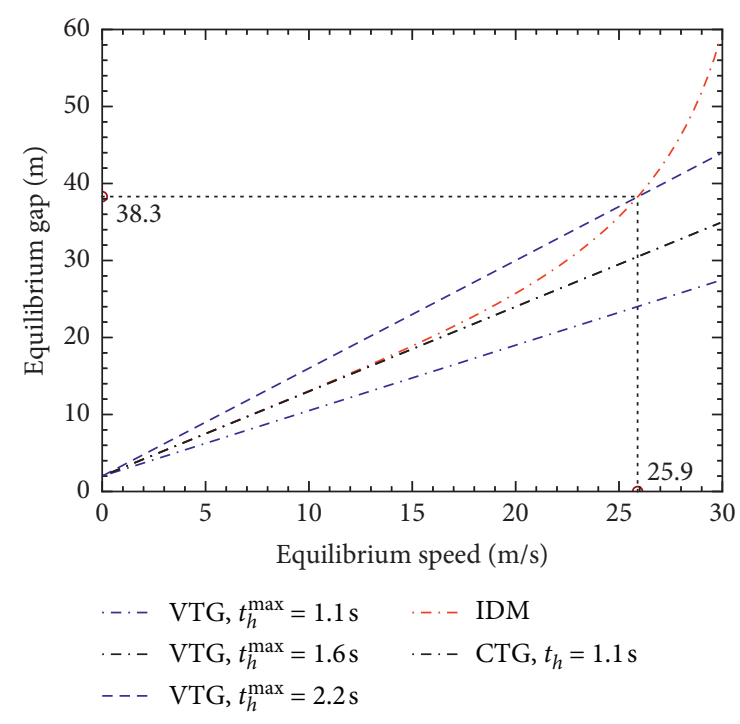

FIGURE 7: Equilibrium speed-gap relation of IDM and ACC vehicles with different desired time gap policies.

policy with $t_{h}^{\max }=2.2 \mathrm{~s}, t_{h}^{\min }=0.6 \mathrm{~s}$, an intersection point (i.e., $v_{e}=25.9 \mathrm{~m} / \mathrm{s}$ ) occurs. It means that when the equilibrium speed is lower than the intersection point, the equilibrium gap of IDM is smaller than the VTG policy, and vice versa. Therefore, reducing the desired time gap of ACC will reduce the distance gap of mixed traffic flow and consequently will increase the throughput of mixed traffic flow.

The distance gap at equilibrium is the sum of the equilibrium gap and vehicle length:

$$
\Delta x_{e}=s_{e}+L
$$

Assuming there are $N$ vehicles in mixed traffic flow, and the penetration of ACC vehicles is $p$, the average distance gap in mixed traffic is calculated as

$$
\Delta \overline{x_{e}}=p \Delta x_{e}^{A}+(1-p) \Delta x_{e}^{M},
$$

where $\Delta x_{e}^{A}=s_{e}^{A}+L$ and $\Delta x_{e}^{M}=s_{e}^{M}+L$ denote the equilibrium distance gap of ACC vehicle and human-driving vehicle, respectively.

The density of mixed traffic can be obtained based on the relation between density and average vehicle distance gap:

$$
k=\frac{1000}{\Delta \overline{x_{e}}} .
$$

Then, the volume can be calculated as

$$
q=v k=\frac{3600 \times v}{\Delta \overline{x_{e}}} .
$$

Figure 8 shows the fundamental diagram of mixed traffic with different desired time gap policies. It is noted that the fundamental diagrams of CTG policy with $t_{h}=1.1 \mathrm{~s}$ (Figure 8(a)) and VTG policy with $t_{h}^{\max }=1.6 \mathrm{~s}, t_{h}^{\min }=0.6 \mathrm{~s}$ (Figure 8(c)) are identical, which is consistent with microscopic fundamental diagram analysis (cf. Figure 7 and equations (18) and (19)). Additionally, it reveals that as the penetration rate of ACC increases, the maximum throughput of mixed traffic flow increases, which indicated that the ACC system can improve the maximum throughput when the desired time gap is set equal to IDM. Figures 8(b)8 (d) show the effect of different desired time gaps on the fundamental diagram of mixed traffic. It is noted that the maximum throughput of mixed traffic flow is gradually reduced with the increase in the desired time gap. Specifically, when the desired time gap of ACC is less than or equal to that of IDM, increasing the penetration of ACC is conducive to improving the throughput of mixed traffic flow (cf. Figures $8(\mathrm{~b})$ and $8(\mathrm{c})$ ). If the desired time gap is larger, however, more ACC vehicles will deteriorate the maximum throughput of mixed traffic flow (cf. Figure 8(d)).

Moreover, an intersection point (density is $23.1 \mathrm{veh} / \mathrm{km}$ ) occurs in Figure 8(d), which is consistent with the previous study [34]. It is noted that when the density is greater than the intersection point, the throughput of the mixed traffic flow with a higher penetration rate of ACC decreases as the density increases. The reason is that when the desired time gap of ACC is larger than that of IDM, there is a demarcation point that determines which equilibrium gap is larger (cf. Figure 7). When the equilibrium gap is smaller than $38.3 \mathrm{~m}$ (i.e., density is greater than $23.1 \mathrm{veh} / \mathrm{km}$ ), the equilibrium gap of ACC is larger than that of IDM. Hence, the high penetration rate of ACC will lessen the throughput of mixed traffic flow. Conversely, if the equilibrium gap is greater than $38.3 \mathrm{~m}$ (i.e., density is smaller than $23.1 \mathrm{veh} / \mathrm{km}$ ), the high penetration rate of ACC will increase the throughput of mixed traffic flow.

4.2. Simulation-Based Investigation of Throughput. To assess the effects of the proposed VTG policy on throughput improvements in mixed traffic flow, simulation experiments are conducted using SUMO [43]. The simulated road section is a hypothetical one-lane with an on-ramp located in the middle of the road section $[32,34]$, as shown in Figure 9. The length of the simulated road section is $6 \mathrm{~km}$ with a speed limit of $120 \mathrm{~km} / \mathrm{h}$ (i.e., $33.33 \mathrm{~m} / \mathrm{s}$ ). The main-line flow varies from 1000 to $3000 \mathrm{veh} / \mathrm{h}$, whereas on-ramp flow varies from 360 to $720 \mathrm{veh} / \mathrm{h}$. In addition, three detectors are placed in the upstream, middle, and downstream of the weaving section, collecting aggregated traffic data every $5 \mathrm{~min}$. The simulation duration is $2 \mathrm{~h}$ with a simulated warm-up time of 30 minutes.

It should be mentioned that the IDM and the ACC validated by PATH have been realized in SUMO. Hence, the proposed VTG policy for ACC vehicles is coded and embed into SUMO. The default gap-acceptance-based lanechanging model in SUMO is selected for merging maneuver. The settings of $t_{h}=1.1 \mathrm{~s}$ and $t_{h}^{\max }=1.6 \mathrm{~s}, t_{h}^{\min }=0.6 \mathrm{~s}$ are used for CTG and VTG policies, respectively. Moreover, the minimum safety distance $s_{0}$ is set to $2 \mathrm{~m}$. The penetration rates $p$ are set to $0 \%, 20 \%, 40 \%, 60 \%, 80 \%$, and $100 \%$ to investigate the effect of penetration rate on throughput in mixed traffic flow. The simulation results are shown in Figure 10. 


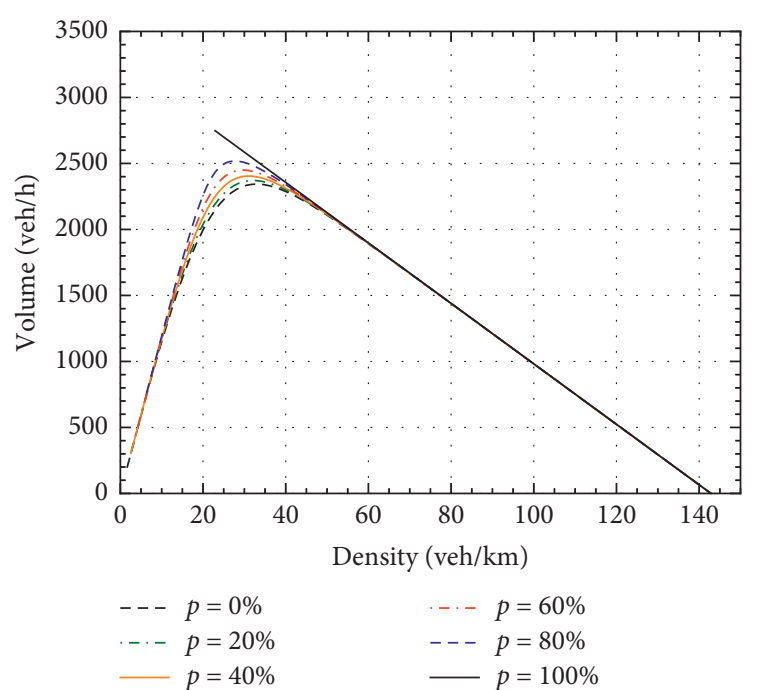

(a)

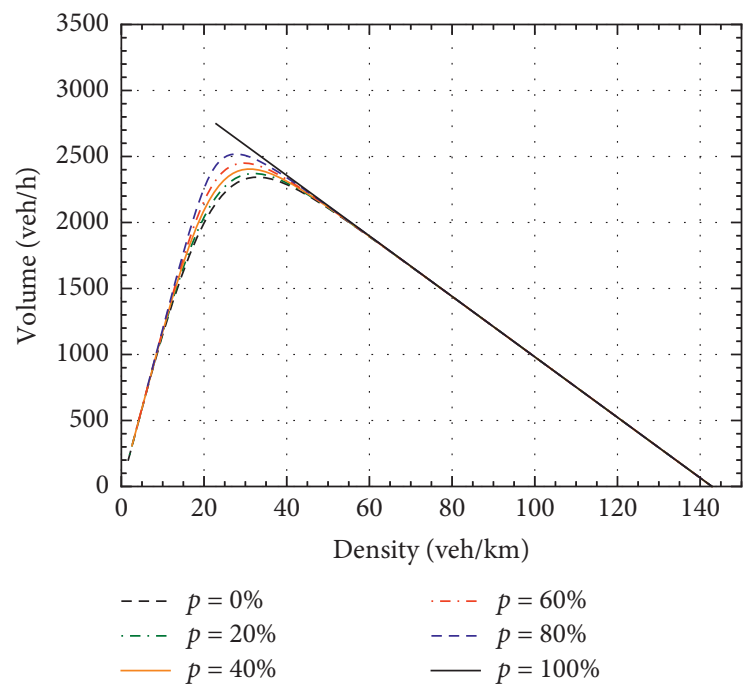

(c)

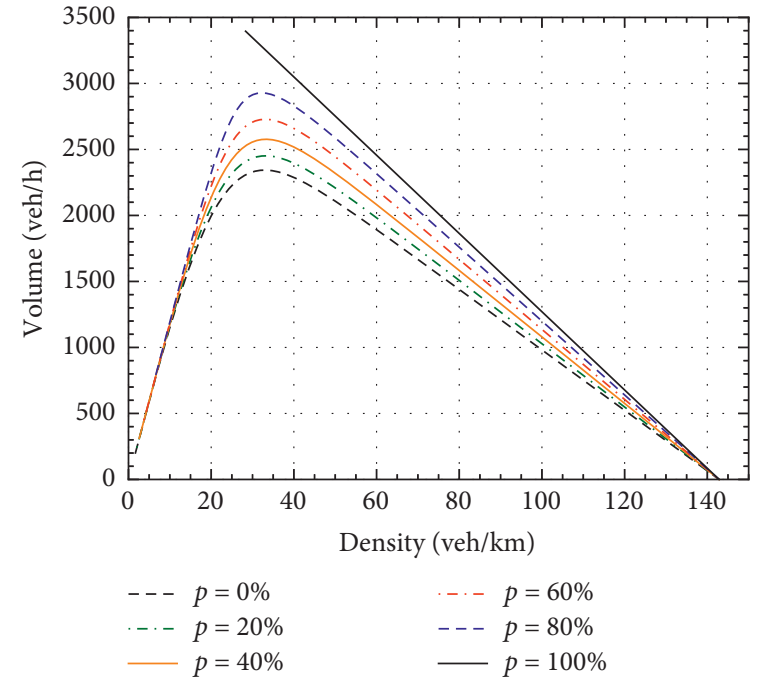

(b)

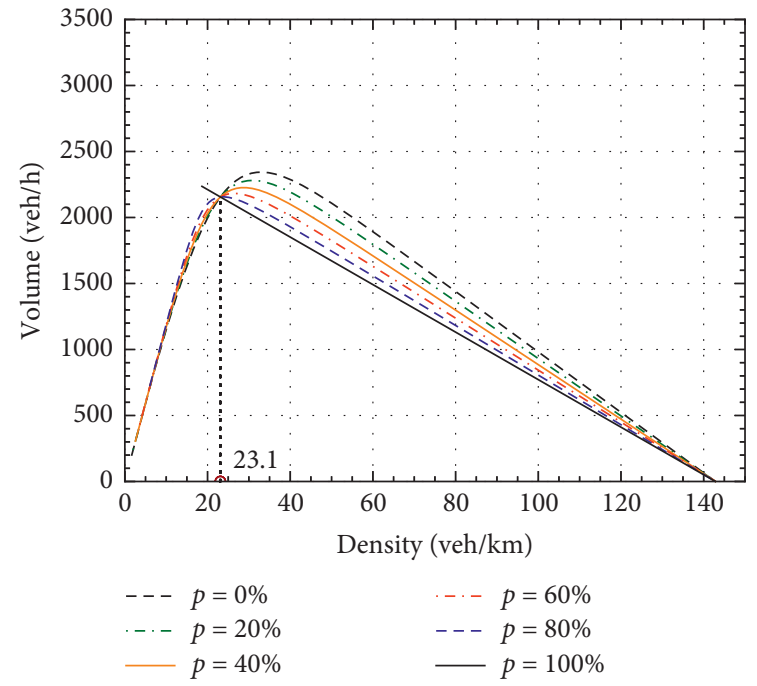

(d)

Figure 8: The fundamental diagram of mixed traffic with different desired time gap policies. (a) CTG policy with $t_{h}=1.1 \mathrm{~s}$. (b) VTG policy with $t_{h}^{\max }=1.1 \mathrm{~s}$ and $t_{h}^{\min }=0.6 \mathrm{~s}$. (c) VTG policy with $t_{h}^{\max }=1.6 \mathrm{~s}$ and $t_{h}^{\min }=0.6 \mathrm{~s}$. (d) VTG policy with $t_{h}^{\max }=2.2 \mathrm{~s}$ and $t_{h}^{\min }=0.6 \mathrm{~s}$.

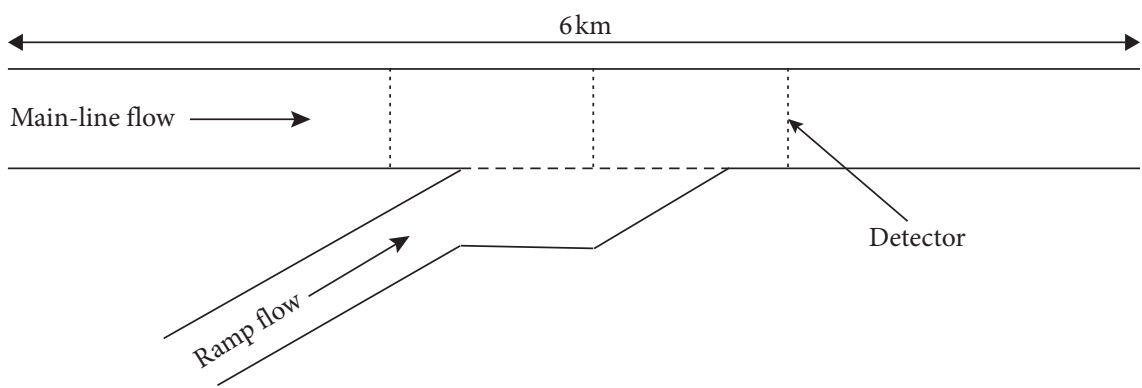

Figure 9: Geometric characteristics of the simulation road segment.

In Figure 10, the blue curve represents the theoretical curves with $0 \%$ penetration rates, and the red curve represents the theoretical curves with the given penetration rates. The black points and red points are the density-volume data of the simulation results with CTG and VTG policy, respectively. It can be clearly seen that there are deviations between the simulation data and the analytical results. This largely results from the fact that the field traffic flow is difficult to reach and maintain at a stable state. However, the corresponding throughput in 


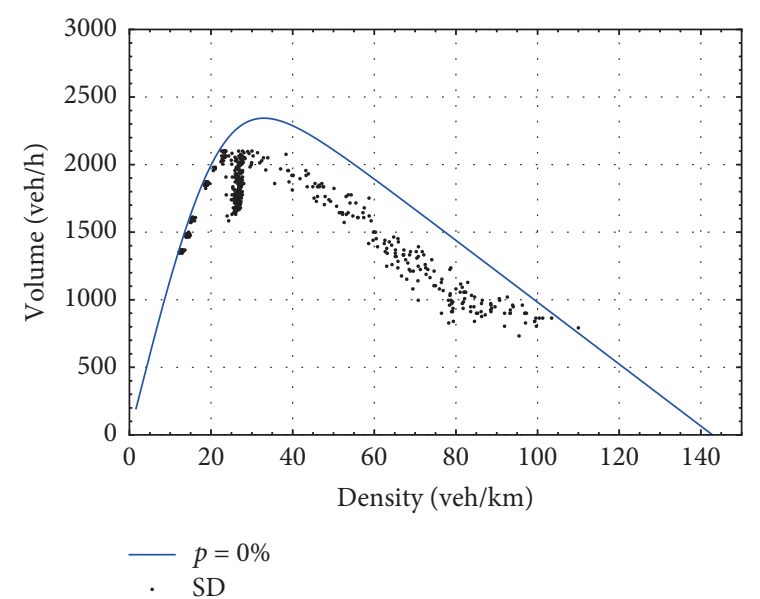

(a)

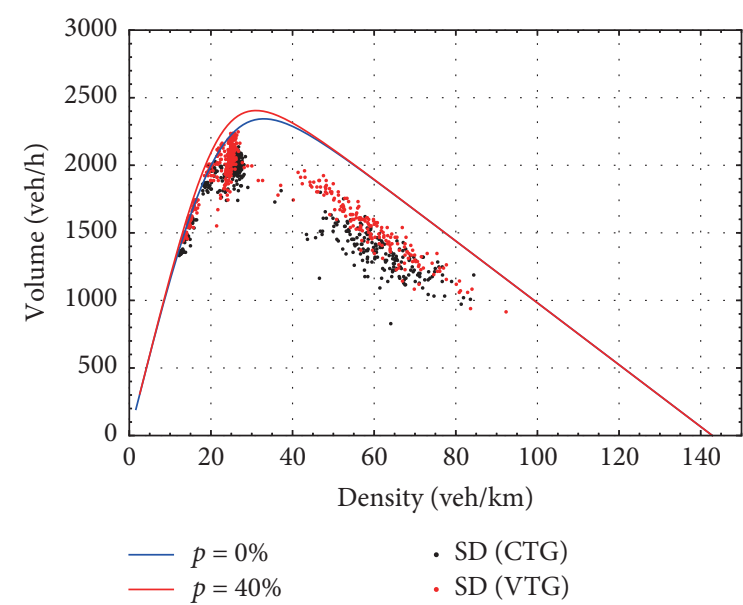

(c)

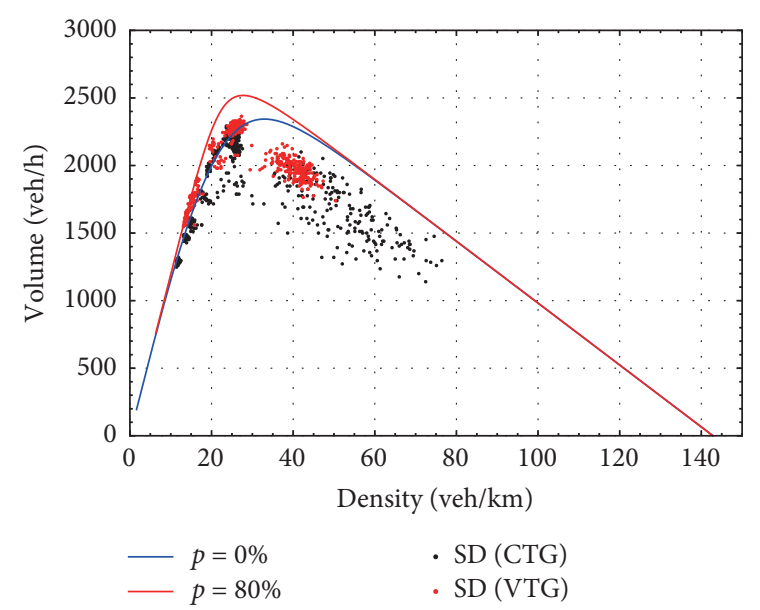

(e)

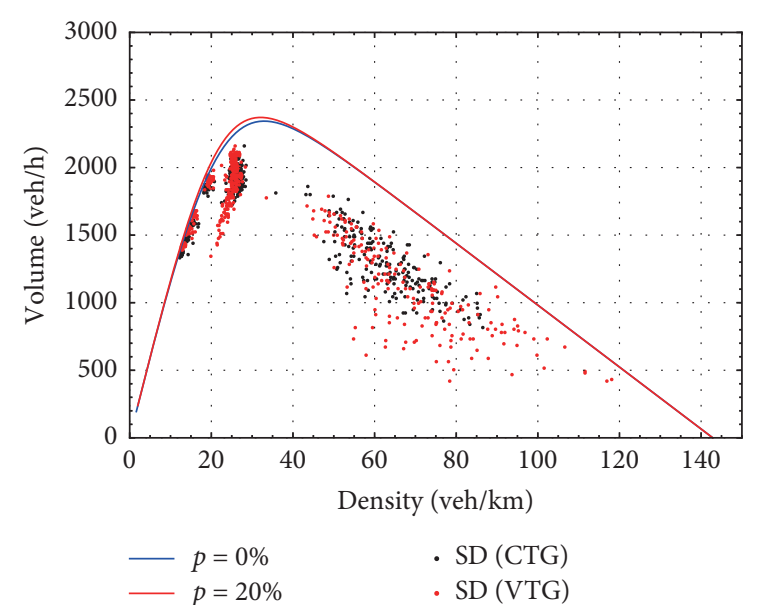

(b)

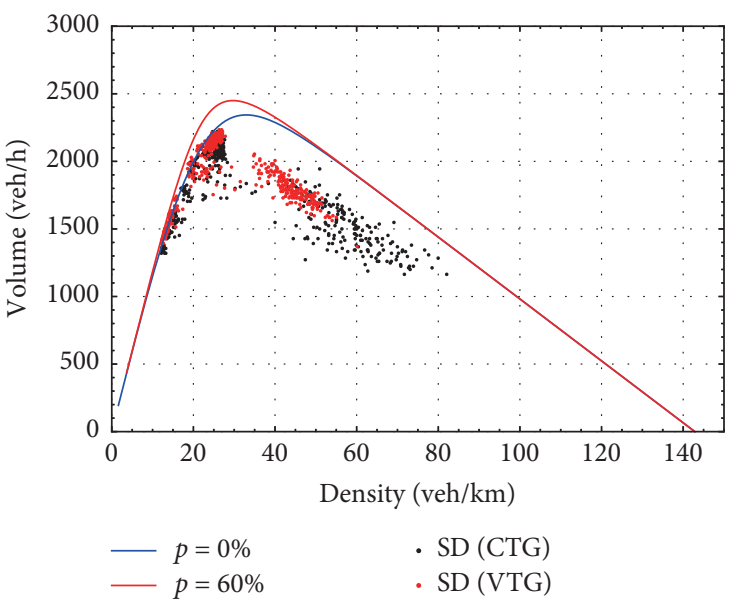

(d)

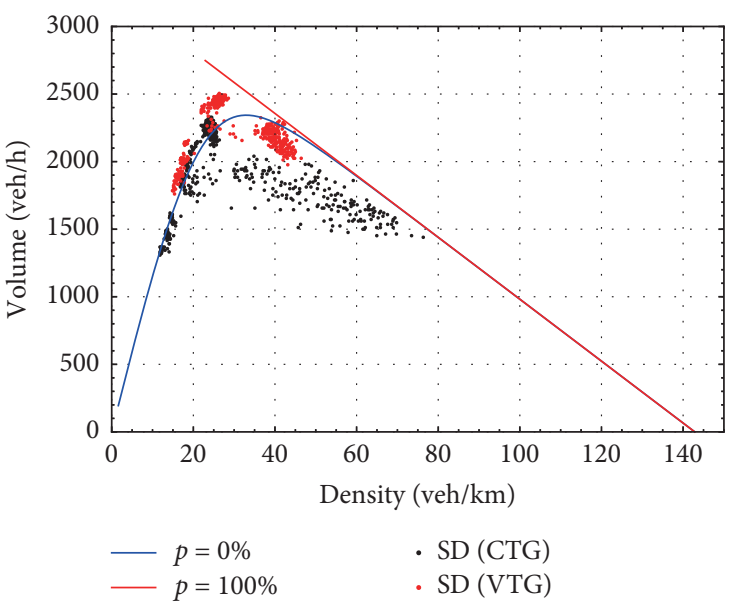

(f)

FIGURE 10: Fundamental diagrams from simulating results of different market penetration rates of ACC vehicles with different desired time gap policies (SD: simulation data).

mixed traffic flow gradually increases with the increase of the penetration rates of ACC vehicles, which is consistent with the analytical results. In addition, it should be noted that the performance with respect to improving throughput in mixed traffic flow with VTG policy is better than that with CTG policy. Meanwhile, the simulation data points of VTG policy gradually cluster in the middle range of density (i.e., $20-40 \mathrm{veh} / \mathrm{km}$ ) with the increase of the penetration rates of ACC vehicles, where the traffic flow operates more efficiently. In summation, the 
simulation results reveal that the proposed VTG policy for ACC vehicles has the potential to improve throughput.

\section{Conclusions}

The aim of the present study is to compare the influence of different time gap policies for ACC on mixed traffic analytically and numerically. First, a modified VTG policy for ACC is illustrated. Then, the stability of mixed traffic flow considering different time gap policies is derived analytically. Analytical results show that the VTG policy is more likely to stabilize the mixed traffic when the equilibrium speed of traffic flow ranges from $5 \mathrm{~m} / \mathrm{s}$ to $25 \mathrm{~m} / \mathrm{s}$. In addition, increasing the maximum desired time gap will enhance the stability of mixed traffic flow, whereas it will reduce the capacity of mixed traffic flow due to a larger inter-vehicle gap. Numerical simulation is also conducted to compare the performance of different time gap policies in suppressing traffic oscillation. Simulation results indicate that it has a better performance in suppressing the magnitude and propagation of oscillation. Finally, the fundamental diagram of the mixed traffic flow is derived and a hypothetical onramp scenario is designed to explore the impact of different time gap policies on the throughput of the bottleneck. Analytical results show that the capacity of mixed traffic flow increases as the penetration rate of ACC vehicles increases. In addition, simulation results show that performance with respect to improving throughput in mixed traffic flow with VTG policy is better than that with CTG policy. Moreover, analytical and simulation-based results demonstrate that the stability analysis procedure is effective. In addition, the results in this study suggest a possibility that the stability of the mixed traffic flow can be used as an intuitive indicator to evaluate the performance of different time gap policies for ACC vehicles in mixed traffic flow in future studies. The findings in this paper offer some important insights into the design of ACC controllers and vehicular traffic control schemes.

It should be mentioned that the conclusions drawn in this paper are based on a classic linear feedback controller for ACC. In the future, more sophisticated control structures for ACC can be used to analyse the effect of ACC on traffic flow following the framework presented in this study. Besides, the practical performance of the proposed VTG policy for ACC vehicles should be tested in the field in future studies.

\section{Data Availability}

The data used to support this study can be obtained from the authors upon request (18114028@bjtu.edu.cn).

\section{Conflicts of Interest}

The authors declare that there are no conflicts of interest regarding the publication of this paper.

\section{Acknowledgments}

This work was supported by the National Key R\&D Program of China (Grant no. 2018YFB1600703) and the National Natural Science Foundation of China (Grant no. 61973028).

\section{References}

[1] Y. Zhou, S. Ahn, M. Chitturi, and D. A. Noyce, "Rolling horizon stochastic optimal control strategy for ACC and CACC under uncertainty," Transportation Research Part C: Emerging Technologies, vol. 83, pp. 61-76, 2017.

[2] V. Milanés and S. E. Shladover, "Modeling cooperative and autonomous adaptive cruise control dynamic responses using experimental data," Transportation Research Part C: Emerging Technologies, vol. 48, pp. 285-300, 2014.

[3] V. Milanes, S. E. Shladover, J. Spring, C. Nowakowski, H. Kawazoe, and M. Nakamura, "Cooperative adaptive cruise control in real traffic situations," IEEE Transactions on Intelligent Transportation Systems, vol. 15, no. 1, pp. 296-305, 2014.

[4] Y. Bian, Y. Zheng, W. Ren, S. E. Li, J. Wang, and K. Li, "Reducing time gap for platooning of connected vehicles via V2V communication," Transportation Research Part C: Emerging Technologies, vol. 102, pp. 87-105, 2019.

[5] R. Rajamani and C. Zhu, "Semi-autonomous adaptive cruise control systems," IEEE Transactions on Vehicular Technology, vol. 51, no. 5, pp. 1186-1192, 2002.

[6] D. Yanakiev and I. Kanellakopoulos, "Nonlinear spacing policies for automated heavy-duty vehicles," IEEE Transactions on Vehicular Technology, vol. 47, no. 4, pp. 1365-1377, 1998.

[7] H. Chehardoli, A. Ghasemi, and A. Najafi, "Centralized and decentralized distributed control of longitudinal vehicular platoons with non-uniform communication topology," Asian Journal of Control, vol. 21, no. 6, pp. 2691-2699, 2019.

[8] A. Ali, G. Garcia, and P. Martinet, "Minimizing the intervehicle distances of the time gap policy for platoon control on highways," in Proceedings of 10th International Conference on Informatics in Control, Automation and Robotics. (ICINCO), pp. 417-424, Reykjavik, Iceland, July 2013.

[9] J. Wang and R. Rajamani, "Should adaptive cruise-control systems be designed to maintain a constant time gap between vehicles?" IEEE Transactions on Vehicular Technology, vol. 53, no. 5, pp. 1480-1490, 2004.

[10] D. Manolis, A. Spiliopulou, F. Vandorou, and M. Papageorgiou, "Real time adaptive cruise control strategy for motorways," Transportation Research Part C: Emerging Technologies, vol. 115, Article ID 102617, 2020.

[11] J. Chen, Y. Zhou, and H. Liang, "Effects of ACC and CACC vehicles on traffic flow based on an improved variable time gap spacing strategy," IET Intelligent Transport Systems, vol. 13, no. 9, pp. 1365-1373, 2019.

[12] C. Wu, Z. Xu, Y. Liu, C. Fu, K. Li, and M. Hu, "Spacing policies for adaptive cruise control: a survey," IEEE Access, vol. 8, no. 99, pp. 50149-50162, 2020.

[13] A. Spiliopulou, D. Manolis, F. Vandorou, and M. Papageorgiou, "Adaptive cruise control operation for improved motorway traffic flow," Transportation Research Record: Journal of the Transportation Research Board, vol. 2672 , no. 22 , pp. 24-35, 2018. 
[14] S. C. Calvert, W. J. Schakel, and J. W. C. van Lint, "Will automated vehicles negatively impact traffic flow?" Journal of Advanced Transportation, vol. 2017, Article ID 3082781, 2017.

[15] M. Seraj, J. C. Li, and T. Z. Qiu, "Expansion of the fundamental diagram from a microscopic multilane modeling framework of mixed traffic," Journal of Advanced Transportation, vol. 2020, Article ID 8878346, 2020.

[16] L. C. Davis, "Effect of adaptive cruise control systems on traffic flow," Physical Review E, vol. 69, no. 6, Article ID 066110, 2004.

[17] A. Kesting, M. Treiber, M. Schönhof, and D. Helbing, "Adaptive cruise control design for active congestion avoidance," Transportation Research Part C: Emerging Technologies, vol. 16, no. 6, pp. 668-683, 2008.

[18] Y. Li, Z. Li, H. Wang, W. Wang, and L. Xing, "Evaluating the safety impact of adaptive cruise control in traffic oscillations on freeways," Accident Analysis \& Prevention, vol. 104, pp. 137-145, 2017.

[19] Y. Li, H. Wang, W. Wang, S. Liu, and Y. Xiang, "Reducing the risk of rear-end collisions with infrastructure-to-vehicle (I2V) integration of variable speed limit control and adaptive cruise control system," Traffic Injury Prevention, vol. 17, no. 6, pp. 597-603, 2016.

[20] Z. Yao, R. Hu, Y. Jiang, and T. Xu, "Stability and safety evaluation of mixed traffic flow with connected automated vehicles on expressways," Journal of Safety Research, vol. 75, pp. 262-274, 2020.

[21] I. Mahdinia, R. Arvin, A. J. Khattak, and A. Ghiasi, "Safety, energy, and emissions impacts of adaptive cruise control and cooperative adaptive cruise control," Transportation Research Record: Journal of the Transportation Research Board, vol. 2674, no. 6, pp. 253-267, 2020.

[22] M. Vajedi and N. L. Azad, "Ecological adaptive cruise controller for plug-in hybrid electric vehicles using nonlinear model predictive control," IEEE Transactions on Intelligent Transportation Systems, vol. 17, no. 1, pp. 113-122, 2016.

[23] C. R. He, J. I. Ge, and G. Orosz, "Fuel efficient connected cruise control for heavy-duty trucks in real traffic," IEEE Transactions on Control Systems Technology, vol. 28, no. 6, pp. 2474-2481, 2020.

[24] K. Jerath and S. N. Brennan, "Analytical prediction of selforganized traffic jams as a function of increasing ACC penetration," IEEE Transactions on Intelligent Transportation Systems, vol. 13, no. 4, pp. 1782-1791, 2012.

[25] M. Michail, K. Mattas, and B. Ciuffo, "Response time and time gap of an adaptive cruise control. An empirical characterization and potential impacts on road capacity," IEEE Transactions on Intelligent Transportation Systems, vol. 21, no. 4, pp. 1677-1686, 2020.

[26] R. E. Wilson, "Mechanisms for spatio-temporal pattern formation in highway traffic models," Philosophical Transactions of the Royal Society A: Mathematical, Physical and Engineering Sciences, vol. 366, no. 1872, pp. 2017-2032, 2008.

[27] G. Orosz, R. E. Wilson, and G. Stepan, "Traffic jams: dynamics and control," Philosophical Transactions of the Royal Society A: Mathematical, Physical and Engineering Sciences, vol. 368, no. 1928, pp. 4455-4479, 2010.

[28] R. E. Wilson and J. A. Ward, "Car-following models: fifty years of linear stability analysis - a mathematical perspective," Transportation Planning and Technology, vol. 34, no. 1, pp. 3-18, 2011.

[29] M. Treiber and A. Kesting, Traffic Flow Dynamics: Data, Models and Simulation, Springer, Berlin, Germany, 2013.
[30] C. Y. Liang and H. Peng, "Optimal adaptive cruise control with guaranteed string stability," Vehicle System Dynamics, vol. 32, no. 4-5, pp. 313-330, 1999.

[31] L. C. Davis, "Stability of adaptive cruise control systems taking account of vehicle response time and delay," Physics Letters A, vol. 376, no. 40-41, pp. 2658-2662, 2012.

[32] A. Talebpour and H. S. Mahmassani, "Influence of connected and autonomous vehicles on traffic flow stability and throughput," Transportation Research Part C: Emerging Technologies, vol. 71, pp. 143-163, 2016.

[33] H. Wang, Y. Qin, W. Wang, and J. Chen, "Stability of CACCmanual heterogeneous vehicular flow with partial CACC performance degrading," Transportmetrica B: Transport Dynamics, vol. 7, no. 1, pp. 788-813, 2019.

[34] Z. H. Yao, R. Hu, Y. Wang et al., "Stability analysis and the fundamental diagram for mixed connected automated and human-driven vehicles," Physica A: Statistical Mechanics and Its Applications, vol. 533, Article ID 121931, 2019.

[35] K. Santhanakrishnan and R. Rajamani, "On spacing policies for highway vehicle automation," IEEE Transactions on Intelligent Transportation Systems, vol. 4, no. 4, pp. 198-204, 2003.

[36] J. Zhou and H. Peng, "Range policy of adaptive cruise control vehicles for improved flow stability and string stability," IEEE Transactions on Intelligent Transportation Systems, vol. 6, no. 2, pp. 229-237, 2005.

[37] S. E. Shladover, D. Su, and X.-Y. Lu, "Impacts of cooperative adaptive cruise control on freeway traffic flow," Transportation Research Record: Journal of the Transportation Research Board, vol. 2324, no. 1, pp. 63-70, 2012.

[38] M. Treiber, A. Hennecke, and D. Helbing, "Congested traffic states in empirical observations and microscopic simulations," Physical Review E, vol. 62, no. 2, pp. 1805-1824, 2000.

[39] J. Sun, Z. Zheng, and J. Sun, "Stability analysis methods and their applicability to car-following models in conventional and connected environments," Transportation Research Part B: Methodological, vol. 109, pp. 212-237, 2018.

[40] J. A. Ward, Heterogeneity, Lane-Changing and Instability in Traffic: A Mathematical Approach, University of Bristol, Bristol, UK, 2009.

[41] S. Gong, J. Shen, and L. Du, "Constrained optimization and distributed computation based car following control of a connected and autonomous vehicle platoon," Transportation Research Part B: Methodological, vol. 94, pp. 314-334, 2016.

[42] Y. Qin and H. Wang, "Analytical framework of string stability of connected and autonomous platoons with electronic throttle angle feedback," Transportmetrica A: Transport Science, vol. 17, no. 1, pp. 59-80, 2021.

[43] P. A. Lopez, W. Evamarie, M. Behrisch et al., "Microscopic traffic simulation using SUMO," in Proceedings of the 2018 21st International Conference on Intelligent Transportation Systems (ITSC), pp. 2575-2582, Maui, HI, USA, November 2018. 